\title{
Managing gout needs more than drugs: 'Il faut le savoir-faire, l'Art et la manière'
}

\section{Frédéric Lioté, ${ }^{1,2,3}$ Hyon Choi ${ }^{4}$}

Gout management has recently been a topic of active discussion, prompted by several European and international recommendations. ${ }^{1-3}$ Nevertheless, a number of studies have reported a suboptimal level of current gout care, including even poorer adherence to prescribed drugs, ${ }^{4}$ than in patients with diabetes or hypertension. ${ }^{5}$ Quality indicators for the treatment of gout developed to date have focused primarily on the use of allopurinol as the most frequently prescribed urate lowering therapy (ULT), adjustment of the maximal dose according to renal function and serum uric acid (SUA) level measurement. ${ }^{67}$

In their provocative, proof-of-concept study, Rees et al ${ }^{8}$ provide important preliminary evidence that treating gout effectively is not just a matter of initiating ULT, but rather of implementing a proper approach that combines patient education, individualised lifestyle advice, and appropriate use of ULTs to achieve and sustain treatment targets (eg, SUA level $<360-300 \mu \mathrm{mol} / \mathrm{l}){ }^{7}$ Over the 1-year trial period, this approach led to more than $90 \%$ of patients achieving the primary treatment target of SUA of $<360 \mu \mathrm{mol} / 1$ recommended by Eular League Against Rheumatism (EULAR), ${ }^{2}$ and to $85 \%$ achieving an SUA of $<300 \mu \mathrm{mol} / 1$, the target level recommended by the British Society for Rheumatology. ${ }^{3}$

Although the trial was an open-label, proof-of-concept study without randomisation or a control group, the effect sizes appear large enough to overcome potential regression to the mean or placebo effects. Furthermore, previous randomised gout

\footnotetext{
1Department of Rheumatology, University Paris Diderot, Paris, France

${ }^{2}$ Service de Rhumatologie, AP-HP, Hôpital Lariboisière, Centre Viggo Petersen, Paris, France

${ }^{3}$ Inserm, UMR 606, Hôpital Lariboisière, Centre Viggo Petersen, Paris, France

${ }^{4}$ Department of Medicine, Section of Rheumatology and the Clinical Epidemiology Unit, Boston University School of Medicine, Boston, Massachusetts, USA
}

Correspondence to Professor Frédéric Lioté, Service de Rhumatologie, AP-HP, Centre Viggo Petersen, Hôpital Lariboisière, 2, rue Ambroise Paré, Paris F-75010,

France; frederic.liote@lrb.aphp.fr trial experience suggests that SUA levels below $6.0 \mathrm{mg} / \mathrm{dl}$ are not attained in placebotreated patients. So these findings do suggest substantial potential benefits from the proposed approach. While the next step of a controlled trial of the approach is currently underway, the proof-of-concept study findings appear instructive in their own right in several ways.

\section{IS GOUT SUCH A DIFFICULT DISEASE TO TREAT?}

Despite the recent publications challenging the quality of current gout care, gout has long been considered potentially 'curable' with a well-characterised pathogenesis and the availability of effective antigout measures. Gout attacks (or acute joint inflammation, if preferred) can result at any time from the deposited urate (monosodium urate; MSU) crystals that form as a consequence of hyperuricaemia; a low grade crystal associated subclinical inflammation occurs continuously. SUA serves as a clear biomarker, which is readily available, affordable and easy to use. With use of ULT options to reduce SUA levels below the crystallisation threshold (eg, lower than $360 \mu \mathrm{mol} / 1$, or even $300 \mu \mathrm{mol} / 1$ in advanced cases), one can reduce gout flares and tophi, ${ }^{910}$ and even heal bone lesions in some patients. ${ }^{11}$ To this effect, the study by Rees et $a l^{8}$ provides key initial evidence that an appropriate comprehensive approach can meaningfully improve gout care, as was long thought possible.

The target-guided approach adopted by the study of Rees et $a l^{8}$ should be viewed analogous to the treat-to-target paradigm in rheumatoid arthritis (RA). In RA, this approach strives for targets, aiming at quick remission, based upon clinical and inflammatory biomarkers and with absence of synovitis by ultrasound examination. A higher goal can be pursued in gout since it is a potentially 'curable' rheumatic disease.

\section{'GO SLOWLY' AND 'GO EFFECTIVELY' TO AVOID THERAPEUTIC INERTIA}

Among ULT options, allopurinol, a xanthine oxidase inhibitor, is the leading choice worldwide. $^{2} 312$ Optimal dosing is guided by renal function to help avoid side effects that range from mild rashes $(\sim 2 \%)$ to rare, but serious hypersensitivity syndromes. While there are some differences of opinion regarding the maximal doses of allopurinol that should be employed, ${ }^{13-15}$ current guideline recommendations suggest that maximal dosages of 800 or $900 \mathrm{mg} /$ day can be safely used if required in patients with normal renal function. $^{2}{ }^{3}$ Alternative available ULT includes febuxostat, another xanthine oxidase inhibitor, ${ }^{12}$ pegloticase (a uricase) and uricosurics such as probenecid, sulfinpyrazone and benzbromarone, while several other potential ULT options are being developed. ${ }^{16} 17$

Allopurinol, when appropriately dosed, is an effective gout treatment as demonstrated by Dutch rheumatologists. ${ }^{16} 17$ Nevertheless, many randomised control trials, mostly conducted in the US gout population, have notably employed the so-called 'usual' allopurinol dosage of $300 \mathrm{mg}$, which appears to be insufficient in many instances. ${ }^{12}$ In a recent survey from UK, ${ }^{16} 44$ out of 164 cases were receiving allopurinol, with $70 \%$ at $300 \mathrm{mg}$ daily, and only 4 (10\%) taking a dose $>300 \mathrm{mg}$ daily. Despite this, $23 \%$ of treated patients had SUA $>360 \mu \mathrm{mol} / 1$, indicating that the therapeutic target was not reached. Renal impairment was the most frequent reason for not escalading allopurinol.

By contrast, in the study of Rees et $a l^{8}$, only $28 \%$ received allopurinol $300 \mathrm{mg}$ daily, while $25 \%$ received $400 \mathrm{mg} /$ day, $25 \% 500 \mathrm{mg} /$ day and $13 \%$ needed higher doses. The median dose was $400 \mathrm{mg}$ daily in the 80 patients taking allopurinol at final visit. Other patients received febuxostat or benzbromarone as alternative ULT after treatment failure. The uptitration of allopurinol was slow and accompanied by close monitoring and patient education. In addition to this leading to the high success rates of meeting the predefined SUA targets, $65 \%$ of patients had fewer gout flares over the 1-year period and a third of the patients had a reduction in number and size of tophi. This 'go slowly' and 'go effectively' approach is consistent with EULAR recommendation \#9 and other studies ${ }^{18}$ to start allopurinol at a low dose (100 mg daily) and increase by $100 \mathrm{mg}$ every 2-4 weeks. ${ }^{2}$ This gradual and stepwise approach also appears to have helped to avoid hypersensitivity skin reactions, ${ }^{18}$ and the gout flares that are well known to follow the initiation of ULT despite the fact that only $4 \%$ of 
patients were given gout flare prophylaxis during the trial. ${ }^{8}$ This challenges current practice recommendations for the need for prophylaxis during initiation of $\mathrm{ULT}^{2}$ and suggests that there is a need for further studies to clarify whether prophylaxis with low dose colchicine or NSAIDs is always really necessary.

This 'go slowly' and 'go effectively' approach would also help to avoid 'therapeutic inertia' in gout management. ${ }^{19}$ Although not widely considered in the gout literature, this phenomenon of therapeutic inertia ${ }^{20}$ has been well known in the management of diabetes for decades, with at least 108 PubMed references relating to its occurrence. In this field, it has been well established that despite the observation of insufficient glycaemic control, physicians do not systematically increase drug treatments, ${ }^{21}$ and this has been attributed to 'clinical inertia'. 22

\section{HOW CAN WE DO BETTER? ARE NEW DRUGS OR MODALITIES THE SOLUTION?}

In this study, Rees et al ${ }^{8}$ evaluated a whole strategy as a 'package deal' that consisted of several components: information, tight monitoring and reassurance. The study has been designed to evaluate the effectiveness of the whole approach, but does not allow one to draw inferences about the effects of individual components of the strategy. Nevertheless, it is entirely conceivable, and even likely, that all the involved components are necessary for the strategy to work. Notably, the management included initial patient education by an experienced specialist, which may be a key to its success. The gout specialist performed arthrocentesis and synovial fluid examination for MSU identification to confirm the diagnosis in all patients. Thus, the 'gold standard'1 was established without a need for additional imaging modalities such as ultrasound. Other notable components included communication with the patient, and taking sufficient time to explain the meaning and significance of hyperuricaemia, in relation to crystal formation, and the clinical manifestations of gout, as well as the importance of treating-to-target when using ULT and the need for treating flares. The online booklet devoted to gout, patient education and regular contacts with nurses also provided additional information to patients.

Overall, these findings suggest that gout can be treated effectively by optimal use of the well established treatment options coupled with better patient education and communication, while there is only a sparing need for the more recently introduced drugs. It remains to be seen whether this approach is feasible in other practice settings, including primary care. ${ }^{7}$ For example, it is unclear whether this approach would be successful if the initial $1 \mathrm{~h}$ consultation with the gout expert and the subsequent follow-up by the trained nurse specialist were substituted by medical and nursing staff in primary care. The potential selection bias acknowledged by the authors calls for future studies with more generalisable patient populations and appropriate comparison groups.

\section{HOW TO ENSURE PATIENT COMIPLIANCE AND PHYSICIAN EDUCATION?}

There are numerous barriers to the implementation of universal quality care involving attitudes and beliefs of physicians and patients. Many physicians think that gout is of little importance, $^{23} 24$ and they are not aware that persistent gout inflammation and association with cardiovascular diseases is an indicator of gout severity. In a small prospective survey on patient and provider expectations in gout, most providers concluded they had adequate skills to teach disease self-management behaviours. $^{25}$ Interestingly, patients requested more information and longer visit times. The latter is in keeping with the study of Rees et $a l^{8}$ and provides evidence that health management should not be reduced to drug prescription, but must also include patient education. Regular phone calls or direct face-to-face meetings with clinical nurses allowed a slow increase of the chosen ULT to achieve the SUA target, while maintaining high compliance rate. ${ }^{8}$ Indeed, diet changes are not so difficult to manage since only few drinks should be totally avoided such as beers, including non-alcoholic beers, high fructose soft drinks, and spirits. High protein, lipid and calories intake should be reduced. Individualised lifestyle and pharmacological management was another notable component that could be evaluated in other studies since busy clinics may be a target setting for this approach. ${ }^{26}$ Overall, however, the direct cost associated with initial monthly monitoring followed by follow-up every 3 months should be readily acceptable.

For physician education, there have been a number of recent national and international recommendations developed on gout management. ${ }^{2} 32728$ Other education modalities have included articles in general or specialty journals, pamphlets, booklets dedicated to patients and physicians, industry sponsored symposia with various size audiences, and head-to-head or small group interactive meetings. However, quality indicators derived from recommendations may not have been satisfactorily reached.

Despite its specialty care setting and lack of control group, the proof-ofconcept study indeed provides strong evidence for its predefined concept that implementing key elements of best practice recommendations can lead to a remarkable success rate in achieving the therapeutic target and maintaining high adherence to ULT over a 12-month period. ${ }^{8}$ We are awaiting replication of these findings in different settings and/or with a longer follow-up. Anyway, the demonstrated considerable benefits appear enough to recommend the same or a similar approach in the current gout management wherever feasible.

Contributors Both authors have contributed to the draft, discussion and final version of the paper.

Funding This work was supported in part by grants from the National Institutes of Health (R01AR056291 and P60AR047785), from Inserm and University Paris Diderot, Sorbonne Paris Cité. The funding sources had no role in the reporting of the study or in the decision to submit the manuscript for publication.

Competing interests FL has received educational grants from several pharmaceutical companies (Novartis Global, Novartis France, Savient, SOBI, Mayoly-Spindler, Ipsen Pharma, Menarini France, Menarini Global), the INSERM and Paris Diderot University, Sorbonne Paris Cité (UFR de médecine) for organising several European Workshops on crystal-induced inflammation and human diseases since 2010; have served on advisory boards fo Novartis Global \& Novartis France, Ipsen Pharma and Menarini France, Mayoly-Spindler, Savient, Astra-Zeneca \& Ardea. HKC has received research funding for other projects from Takeda Pharmaceuticals and Savient Pharmaceuticals, has served on advisory boards for Takeda Pharmaceuticals, Savient Pharmaceuticals, Novartis, AstraZeneca, and URL Pharma.

Provenance and peer review Commissioned; externally peer reviewed.

Received 4 January 2013

Accepted 19 March 2013

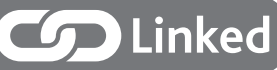

- http://dx.doi.org/10.1136/annrheumdis-2012-201676

Ann Rheum Dis 2013;72:791-793.

doi:10.1136/annrheumdis-2013-203226

\section{REFERENCES}

1. Zhang W, Doherty M, Pascual E, et al. EULAR evidence based recommendations for gout. Part I: Diagnosis. Report of a taskforce of the Standing Committee for International Clinical Studies Including Therapeutics (ESCISIT). Ann Rheum Dis 2006;65:1301-11. 
2. Zhang W, Doherty M, Pascual E, et al. EULAR evidence based recommendations for gout. Part I: Diagnosis. Report of a taskforce of the Standing Committee for International Clinical Studies Including Therapeutics (ESCISIT). Ann Rheum Dis 2006;65:1312-24.

3. Jordan KM, Cameron JS, Snaith M, et al. British Society for Rheumatology and British Health Professionals in Rheumatology guideline for the management of gout. Rheumatology (Oxford) 2007; 46:1372-4

4. Reach G. Treatment adherence in patients with gout. Joint Bone Spine 2011;78:456-9.

5. Briesacher BA, Andrade SE, Fouayzi $\mathrm{H}$, et al. Comparison of drug adherence rates among patients with seven different medical conditions. Pharmacotherapy 2008;28:437-43.

6. Singh JA, Alpert MD, Kerr G. A national survey of Veterans Affairs rheumatologists for relevance of quality of care indicators for gout management. Arthritis Care Res (Hoboken) 2010;62:1306-11.

7. Singh JA, Hodges JS, Toscano JP, et al. Quality of care for gout in the US needs improvement. Arthritis Rheum 2007;57:822-9.

8. Rees F, Jenkins W, Doherty M. Patients with gout adhere to curative treatment if informed appropriately: proof-of-concept observational study. Ann Rheum Dis 2013; 12:826-30.

9. Perez-Ruiz F, Calabozo M, Pijoan Jl, et al. Effect of urate-lowering therapy on the velocity of size reduction of tophi in chronic gout. Arthritis Rheum 2002;47:356-60.

10. Perez-Ruiz F, Lioté F. Lowering serum uric acid levels: what is the optimal target for improving clinical outcomes in gout? Arthritis Rheum 2007:57:1324-8.

11. Baraf HS, Matsumoto AK, Maroli AN, et al. Resolution of gouty tophi after twelve weeks of pegloticase treatment. Arthritis Rheum 2008;58:3632-4.

12. Jansen TL, Richette P, Perez-Ruiz F, et al International position paper on febuxostat. Clin Rheumatol 2010:29:835-40.

13. Vázquez-Mellado J, Morales EM Pacheco-Tena C, et al. Relation between adverse events associated with allopurinol and renal function in patients with gout. Ann Rheum Dis 2001;60 981-3.

14. Dalbeth N, Kumar S, Stamp L, et al. Dose adjustment of allopurinol according to creatinine clearance does not provide adequate control of hyperuricemia in patients with gout. $J$ Rheumatol 2006;33:1646-50.

15. Stamp LK, O'Donnell JL, Zhang $\mathrm{M}$, et al. Using allopurinol above the dose based on creatinine clearance is effective and safe in patients with chronic gout, including those with renal impairment. Arthritis Rheum 2011;63:412-21.

16. Reinders MK, van Roon EN, Houtman PM, et al. Biochemical effectiveness of allopurinol and allopurinol-probenecid in previously benzbromarone-treated gout patients. Clin Rheumatol 2007:26:1459-65.

17. Reinders MK, van Roon EN, Jansen $\mathrm{TL}$, et al. Efficacy and tolerability of urate-lowering drugs in gout: a randomised controlled trial of benzbromarone versus probenecid after failure of allopurinol. Ann Rheum Dis 2009;68:51-6.

18. Stamp LK, Taylor WJ, Jones PB, et al. Starting dose is a risk factor for allopurinol hypersensitivity syndrome: a proposed safe starting dose of allopurinol. Arthritis Rheum 2012;64:2529-36.

19. Roddy E, Zhang W, Doherty M. Concordance of the management of chronic gout in a UK primary-care population with the EULAR gout recommendations. Ann Rheum Dis 2007;66:1311-15.
20. Mohan AV, Phillips LS. Clinical inertia and uncertainty in medicine. JAMA 2011;306:383; author reply 383-4.

21. Simon $\mathbf{D}$. Therapeutic inertia in type 2 diabetes: insights from the PANORAMA study in France. Diabetes Metab 2012;38(Suppl 3): S47-52.

22. Avignon A, Attali C, Sultan A, et al. Clinical inertia: viewpoints of general practitioners and diabetologists. Diabetes Metab 2012;38(Suppl 3): S53-8.

23. Spencer K, Carr A, Doherty M. Patient and provider barriers to effective management of gout in general practice: a qualitative study. Ann Rheum Dis 2012;71:1490-5.

24. Doherty M, Jansen TL, Nuki G, et al. Gout: why is this curable disease so seldom cured? Ann Rheum Dis 2012;71:1765-70.

25. Harrold LR, Mazor KM, Velten S, et al. Patients and providers view gout differently: a qualitative study. Chronic IIIn 2010;6:263-71.

26. Benhamou PY. Improving diabetes management with electronic health records and patients' health records. Diabetes Metab 2011;37(Suppl 4): S53-6.

27. Khanna D, Khanna PP, Fitzgerald JD, et al. 2012 American College of Rheumatology guidelines for management of gout. Part 2: therapy and antiinflammatory prophylaxis of acute gouty arthritis. Arthritis Care Res(Hoboken) 2012;64 1447-61.

28. Khanna D, Fitzgerald JD, Khanna PP, et al. 2012 American College of Rheumatology guidelines for management of gout. Part 1: systematic nonpharmacologic and pharmacologic therapeutic approaches to hyperuricemia. Arthritis Care Res (Hoboken) 2012;64:1431-46. 


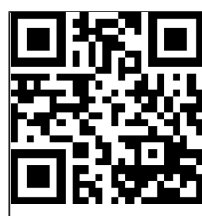

Editor's choice Scan to access more free content
Academic Rheumatology, University of Nottingham, Nottingham, UK

\section{Correspondence to}

Michael Doherty, Academic Rheumatology, University of Nottingham, Clinical Sciences Building, City Hospital, Nottingham NG5 1PB, UK: michael.doherty@nottingham. ac.uk

Accepted 19 April 2012 Published Online First 7 June 2012

\title{
Patients with gout adhere to curative treatment if informed appropriately: proof-of-concept observational study
}

\author{
Frances Rees, Wendy Jenkins, M Doherty
}

\begin{abstract}
Introduction Many doctors believe that patients with gout are unwilling to receive urate-lowering therapy (ULT) and blame them for poor adherence to management. Objective To test the effectiveness of a complex intervention for gout that incorporates key elements of current guidelines, including full patient information, delivered in an optimal setting (specialist hospital clinic). Method Observational study of patients reporting ongoing attacks of gout recruited from primary care lists. 106 participants (94 men, 12 women; mean age 61 years) were enrolled in the study. Patients received a predominantly nurse-delivered intervention that included education, individualised lifestyle advice and appropriate ULT. The predefined goal was to achieve serum uric acid (SUA) levels $\leq 360 \mu \mathrm{mol} / \mathrm{l}$ after 1 year in at least $70 \%$ of participants.
\end{abstract}

Results Of the 106 participants at baseline, 16\% had tophi; mean (SD) baseline SUA was 456 (98) $\mu \mathrm{mol} / \mathrm{l}$. All participants agreed to joint aspiration to confirm gout and all wished to receive ULT. At 12 months, $92 \%$ of the 106 participants had achieved the therapeutic target (SUA $\leq 360 \mu \mathrm{mol}$ ); $85 \%$ had SUA $<300 \mu \mathrm{mol} / \mathrm{l}$. Allopurinol was the most commonly used ULT, requiring a median dose of $400 \mathrm{mg}$ daily to achieve the target. Improvements in Short Form-36 were observed (significant for pain) after 1 year.

Conclusion A predominantly nurse-led intervention including education, lifestyle advice and ULT can successfully achieve the recommended treatment target in more than 9 out of 10 patients. Full explanation and discussion about the nature of gout and its treatment options and individualisation of management probably account for this success.

\section{INTRODUCTION}

Gout is a crystal deposition disease that affects $1-2 \%$ of UK adults, increasing in prevalence with age to affect $7 \%$ of men aged $>65$ and $3 \%$ of women aged $>85 .{ }^{1}$ It is the most common inflammatory arthritis in men and the most common inflammatory arthritis in older women. ${ }^{1-3}$ Unlike other common forms of arthritis we have a good understanding of gout pathogenesis and have effective treatments to eliminate the causative agent (urate crystals) and 'cure' the disease. ${ }^{4}$

Unfortunately, audit shows that the management of patients with gout is far from optimal. . $^{5-7}$ Three UK studies ${ }^{57}$ suggest that only one-third to one-half of patients with gout receive urate lowering therapy (ULT) and that even when given, ULT is often prescribed at a single fixed dose (usually allopurinol $300 \mathrm{mg}$ daily) that is insufficient for many patients. ${ }^{7}$ Furthermore, few patients receive a clear explanation of their gout or appropriate lifestyle advice to reduce predisposing risk factors. ${ }^{7}$ As a consequence, only a minority become free from gout, the majority continuing to experience acute attacks and to be at risk of progression of their disease and of developing secondary irreversible joint damage. ${ }^{3}$ There are many barriers to care of gout ${ }^{7}$ but the commonly documented poor adherence to ULT is often blamed more on patients than a lack of appropriate information from their doctor. ${ }^{10} 11$

Both the British Society for Rheumatology and European League Against Rheumatism have published evidence-based recommendations for management of gout. ${ }^{12} 13$ However, these are based largely on expert consensus, supported by relatively limited research evidence that mainly focuses on short-term studies of individual treatments. To our knowledge there are no long-term trials investigating the benefits of a complex intervention or 'package of care' that reflects recommended best practice. Notwithstanding the absence of randomised controlled trial evidence, there is strong consensus on the components of management that will eliminate urate crystals and effect a 'cure'. ${ }^{14}$ Specifically, this is to combine patient education and individualised lifestyle advice with appropriate use of ULTs to achieve and sustain a target serum uric acid (SUA) level of <360-300 $\mu \mathrm{mol} / 1.91113$

Because of the high prevalence of gout and ready availability of effective treatment the vast majority of patients with gout are managed in primary care. Nurse delivery of care that reflects recommended best practice is a model that has been applied successfully to management of other common chronic conditions such as asthma ${ }^{15}$ and diabetes. ${ }^{16}$ Therefore the aim of this study was to test the effectiveness of a package of care for patients with gout that (1) incorporates the key elements of current recommended 'best practice'12 13; (2) is predominantly delivered by a nurse; (3) is delivered in the optimal setting of an expert, hospital-based gout clinic. Should this 'proof-of-concept' study be successful, the package of care would next be tested in a general practice setting (funded, as for this proof-of-concept study, by Arthritis Research UK). 


\section{METHODS}

Approval for the study was obtained from the National Health Service (NHS) Nottingham County research ethics committee.

\section{Participants}

Participants were recruited from 25 general practices in Nottinghamshire. Patients with a diagnosis of gout on the general practice registers were eligible if they were aged between 30 and 100, had a definite diagnosis of gout ${ }^{17}$ and reported at least one acute gout attack within the previous year, irrespective of any ULT. Potentially eligible participants were first identified through a postal questionnaire to patients with gout on the practice registers. Respondents reporting attacks within the past 12 months were telephoned for a brief discussion to confirm likely eligibility, and then sent full written information about the study and invited for clinical assessment in Academic Rheumatology, City Hospital, where fully informed written consent was obtained. The recruitment period ran from March to July 2010.

\section{Intervention}

At their first visit each participant received a full clinical assessment (enquiry and examination) from a rheumatologist specialising in crystal-associated arthritis (MD). The site and size of any subcutaneous tophi was noted. All participants were offered aspiration preferentially of an intercritical joint (mainly first metatarsophalangeal joint or knee), or a tophus, but a minority preferred aspiration of a previously unaffected knee to an intercritical small joint, to confirm definite 'crystal-proven' gout. ${ }^{17}$ Synovial fluid crystal identification was undertaken in Academic Rheumatology (by MD) and the participant informed of the result within $10 \mathrm{~min}$ of aspiration.

Participants with definite gout ${ }^{17}$ were given detailed information and education about (1) the cause of the disease; (2) its recognised risk factors; (3) clinical consequences with respect to ongoing acute attacks and possible irreversible joint damage; (4) strategies, including discussion of individualised modifiable risk factors (such as weight loss if obese) and ULT, to sufficiently reduce SUA levels below the critical 'saturation point' of 360 $\mu \mathrm{mol} / \mathrm{l}$ to prevent new crystal formation and to dissolve existing crystals (ie, to effect a 'cure'). Illness perceptions about the causes of their gout, its effect on their life, the likely outcome of their gout and available treatment options were fully discussed and an individualised management plan was agreed. An Arthritis Research UK information leaflet ${ }^{18}$ on gout was also given to all participants. Cardiovascular risks from hyperuricaemia were only dealt with in those patients who specifically enquired, since this remains a controversial topic and is not an indication for ULT. The initial interview, assessment, discussion and agreement of a management plan with the doctor took approximately $1 \mathrm{~h}$, twice that of a routine NHS new patient appointment, but did include joint aspiration and crystal analysis. Blood was taken for measurement of baseline SUA and renal function by a specialist gout nurse (WJ), who also reinforced the management plan, gave the participant the written information and booked the follow-up visit (approximately $20 \mathrm{~min}$ ). Other blood tests and joint radiographs were performed only if clinically indicated.

Participants were subsequently followed up by the specialist nurse by telephone or in person to monitor clinical progress and success of lifestyle modification, to titrate up ULT according to SUA levels, to adjust the management plan and to deal with concerns as they arose. The frequency of nurse appointments and repeat blood tests was determined by individual needs, although
SUA measuring and upward titration of ULT was approximately monthly until the therapeutic target was reached, then SUA was measured every 3 months. The nurse-led the management but could consult the rheumatologist to discuss certain clinical decisions (eg, change from one ULT to another). Follow-up was for 1 year. The final nurse-led visit lasted approximately $20 \mathrm{~min}$.

\section{Outcome measures}

The primary outcome was the percentage of patients who had their SUA reduced below $360 \mu \mathrm{mol} / \mathrm{l}$ at 12 months-success was defined empirically as $70 \%$ achieving this therapeutic target at 12 months. Other outcomes were the percentage achieving a SUA of $<300 \mu \mathrm{mol} / 1$ at 12 months; frequency of acute attacks; number and size of tophi; and the Short Form-36 (SF-36) qualityof-life measure (performed at baseline and 12 months).

\section{Statistics}

Descriptive statistics were used to determine the percentage of patients in whom the therapeutic target was achieved. This was analysed on an intention-to-treat (ITT) basis (last value carried forward). Change in SF-36 was analysed with a Student t test, pairing questionnaires at baseline and final visit in study completers, using SPSS version 14.0.

\section{RESULTS}

\section{Participant characteristics and adherence}

The response rate to the questionnaire was $55 \%$. Twenty-five responders were considered ineligible or declined involvement at the telephone interview but 116 were considered potentially eligible and attended Academic Rheumatology. Of these, 106 participants met the eligibility criteria (eight had calcium pyrophosphate crystal deposition and two had uncomplicated osteoarthritis) and proceeded to the study. Table 1 illustrates the baseline characteristics of the study participants.

At their initial visit, after a full explanation of gout, all participants consented to intercritical aspiration of a joint to confirm gout and all wished to receive ULT. Adherence to treatment was good with 96 participants (91\%) completing the 12 -month follow-up. Reasons for non-completion were death due to nontreatment-related causes (two patients); withdrawal due to personal time constraints (one); withdrawal due to side effects of ULT (three); lost to follow-up (three); and one participant on the renal transplantation list was advised not to start ULT but to await review after he had received his transplant (which occurred after study completion). Side effects experienced were diarrhoea, headaches and dizziness with benzbromarone and a rash and gastrointestinal upset with allopurinol.

At the final visit (ITT) 80 participants receiving ULT (79\%) were taking allopurinol (median dose $400 \mathrm{mg}$ daily; range 100$700 \mathrm{mg} ; 100 \mathrm{mg}$ (one patient), $200 \mathrm{mg}$ (six), $300 \mathrm{mg}$ (22), $400 \mathrm{mg}$ (20), $500 \mathrm{mg}$ (20), $600 \mathrm{mg}$ (nine), $700 \mathrm{mg}$ (two)); 16 (16\%) were taking febuxostat (80 mg (11), $120 \mathrm{mg}$ (five)); and five (5\%) were taking benzbromarone (50 mg (two), $100 \mathrm{mg}$ (three)). Reasons for switching from allopurinol to second-line ULT were treatment failure (eight), side effects (11) or concomitant drug interaction (two).

\section{Primary outcome}

At study end the percentage of participants with a SUA $<360$ $\mu \mathrm{mol} / \mathrm{l}$ was $92 \%$ (ITT analysis). This was considerably greater than the predefined target of $70 \%$. Eighty-five per cent of participants had a SUA $<300 \mu \mathrm{mol} / 1$ at 12 months. 
Table 1 Baseline characteristics

\begin{tabular}{lc}
\hline Characteristics & Value \\
\hline Total number of participants & 106 \\
Number of men (\%) & $94(89)$ \\
Age (years), mean (SD) & $61(11)$ \\
Gout: & $14(13)$ \\
$\quad$ Duration (years), mean (SD) & 100 \\
$\quad$ Acute attacks ever (\%) & 46 \\
$\quad$ Chronic joint symptoms (\%) & $17(16)$ \\
Tophi: & \\
$\quad$ Clinically evident tophi at baseline (\%) & $65(12)$ \\
Renal function: & $102(20)$ \\
Baseline eGFR (ml/min/1.73 m²), mean (SD) & \\
$\quad$ Baseline creatinine ( $\mu$ mol/l), mean (SD) & $4(4)$ \\
Attacks: & \\
$\quad$ Number of attacks in year before study, mean (SD) & $456(98)$ \\
SUA: & $509(160-716)$ \\
Baseline SUA ( $\mu$ mol/l), mean (SD) & $14(13)$ \\
SUA ( $\mu$ mol/l), median (range) & $5(5)$ \\
$\quad$ Number (\%) with baseline SUA <360 $\mu$ mol/l \\
$\quad$ Number (\%) with baseline SUA <300 $\mu$ mol/l \\
ULT: \\
$\quad$ Number receiving ULT at baseline (\%) \\
$\quad$ Allopurinol \\
$\quad$ Sulphinpyrazone & $28(26)$ \\
Duration ULT (years), mean (SD) & 27 \\
Comorbidities number (\%): & 1 \\
Hypertension & $5(7)$ \\
Renal impairment (eGFR <60 ml/min/1.73 m²) & $32(30)$ \\
Hyperlipidaemia & $21(20)$ \\
Ischaemic heart disease & $12(11)$ \\
Diabetes & $11(10)$ \\
\hline
\end{tabular}

eGFR, estimated glomerular filtration rate; SUA, serum uric acid; ULT, urate-lowering therapy.

\section{Secondary outcomes}

Participants had a mean of eight SUA measurements during the observation period. The mean SUA reduced from baseline to 268 $\mu \mathrm{mol} / \mathrm{l}$ (table 2). In the 17 patients with tophi at baseline almost one-third had a reduction in number or size at final visit.

In study completers, the mean number of self-reported attacks/ year reduced to 2.4 (SD 2.3). Table 3 shows the decline in the number of attacks and the number of participants experiencing attacks with time.

After full discussion, only four participants (4\%) opted for prophylaxis (colchicine $0.5 \mathrm{mg}$ twice daily) during upward titration of ULT. These four participants had a mean of nine attacks during the follow-up period (SD 2.9). This is compared with a mean of 2.4 (SD 2.3) attacks for the whole group. Comparison of SF-36 scores in study completers shows that there was a statistically significant improvement in the bodily pain domain $(p=0.016)$.

\section{DISCUSSION}

This study shows that with a 'package of care' that includes patient education, individualised lifestyle advice and slow upward titration of ULT according to serial SUA levels (ie, recommended best practice), ${ }^{12} 13$ more than $90 \%$ of participants achieved the therapeutic target of a SUA $<360 \mu \mathrm{mol} / 1$, with $85 \%$ achieving a SUA $<300 \mu \mathrm{mol} / 1$. After a full explanation about the cause of gout, its risk factors and prognosis (including the risk of chronic joint damage), and available treatment strategies that can eliminate the crystals, all participants wished to receive ULT. There was good adherence to treatment, with more than $90 \%$ of participants completing the 12-month observation period.
Given optimal circumstances (patient education, patients knowing their therapeutic target and regular contact with a nurse specialist), the recommended complex intervention is effective in more than nine out of 10 people with gout.

There are no previous studies of complex interventions in gout. However, there are studies that show poor adherence to ULT10 11 19-indeed, adherence to ULT for gout is possibly the worst of any medication for chronic disease. ${ }^{11} 19$ The need for patient and professional education to enable 'cure' in gout has been highlighted previously. ${ }^{20}$ However, despite international guidelines published in $2006,{ }^{13}$ significant barriers to effective gout management remain. ${ }^{21} 22$ A recent study in this department found that the main barriers to treatment were a lack of understanding of both the aetiology and management of gout in men and women with gout but also in health professionals. ${ }^{21}$ Many people focus mainly on acute attacks and have no concept of ongoing, potentially damaging crystal deposition that may lead to tophi and irreversible joint damage. ${ }^{21}$ When ULT is offered, it is often given as a single efficient dose, which commonly provokes acute attacks and, without a clear explanation, patients often stop the ULT and are disinclined to restart it. ${ }^{21}$ However, as in this study, if ULT is titrated slowly and the risk of flare is explained to patients they then understand the need to continue their medication despite flaring and the need for long-term treatment to maintain cure. This suggests that education of both patients and health professionals is of paramount importance if both recommended best practice and good adherence are to be achieved. With full explanation, dealing with illness perceptions and barriers to treatment, adherence can be achieved that is even higher than that for other chronic diseases. ${ }^{23}$ Of interest, every patient in this study accepted an intercritical joint aspirate to confirm with $100 \%$ confidence whether they had gout. This is much higher than many people would have predicted, but again reflects the importance of a full explanation in determining patient decision-making.

Most patients were taking allopurinol at study completion, confirming that this is a well-tolerated and effective ULT to be considered first for patients with gout. ${ }^{12} 1324$ However, the median dose of allopurinol required to achieve the therapeutic target was $400 \mathrm{mg}$. This is in accord with a previous community study in Nottingham, ${ }^{7}$ which found that many patients who receive the commonly prescribed single dose of $300 \mathrm{mg}$ dose are undertreated and continue to have acute attacks and progression of their disease. This reinforces the requirement to individualise the dose of ULT by titration against the SUA until the therapeutic target has been achieved. ${ }^{12}{ }^{13}$ This, in conjunction with full explanation, also accounts for the high success rate in achieving the therapeutic target, in contrast to recent large randomised control trials that report low success rates when using a fixed-dose regimen of allopurinol $300 \mathrm{mg}$ daily. ${ }^{25} 26$ The majority of patients who had a contraindication or side effects with allopurinol were successfully treated with a second or third ULT, with only three participants leaving the study having experienced self-limiting but troublesome side effects from ULT and being unwilling to try alternative options.

After full discussion most patients expressed a preference for no prophylaxis during ULT dose escalation, especially with the slow titration that is possible with allopurinol. Interestingly, despite this, the mean number of attacks during the 12-month observation period was less than that reported in the previous year. This suggests that unlike initiation with $300 \mathrm{mg}$ allopurinol or $80 \mathrm{mg}$ febuxostat (both very efficient urate-lowering doses which usually provoke acute attacks and therefore justify consideration of prophylaxis), ${ }^{12}{ }^{13}$ a regimen with a low starting 
Table 2 Characteristics at final visit (intention to treat)

\begin{tabular}{lc}
\hline Characteristics & Total (106) \\
\hline SUA at final visit & \\
$\quad$ Final visit SUA $(\mu \mathrm{mol} / /)$, mean (SD) & $268(68)$ \\
$\quad$ Final visit SUA $(\mu \mathrm{mol} / /)$, median (range) & $288(130-563)$ \\
$\quad$ Number with uric acid $<360 \mu \mathrm{mol} / /(\%)$ & $98(92)$ \\
$\quad$ Number with uric acid $<300 \mu \mathrm{mol} / /(\%)$ & $90(85)$ \\
Tophi & \\
$\begin{array}{l}\text { Of } 17 \text { patients with tophi at baseline, number with reduced size/ } \\
\text { number at final visit (\%) }\end{array}$ & $5(29)$ \\
\hline
\end{tabular}

SUA, serum uric acid.

Table 3 Frequency of attacks per quarter

\begin{tabular}{lllll}
\hline Time & $\mathbf{0 - 3}$ Months & $\mathbf{3 - 6}$ Months & $\mathbf{6 - 9}$ Months & $\mathbf{9 - 1 2}$ Months \\
\hline No of attacks & 68 & 61 & 49 & 35 \\
No of people & 43 & 38 & 31 & 28
\end{tabular}

Sixty-five per cent reported fewer attacks than the previous year, $13 \%$ reported the same number and $22 \%$ reported more attacks (mean 2.7 more attacks than the previous year). The group with more attacks had a higher mean baseline serum uric acid compared with the whole group (482 (92) vs 456 (98) $\mu \mathrm{mol} / \mathrm{l}$ ) and a greater percentage had tophi at baseline (33\% vs $16 \%)$.

dose of ULT (eg, $100 \mathrm{mg}$ allopurinol or $50 \mathrm{mg}$ benzbromarone) and subsequent monthly upward titration may not cause more attacks than the patient might otherwise have experienced. Therefore, the use of a second drug (colchicine or a non-steroidal anti-inflammatory drug) with its own potential for side effects may not be required for this indication. The caveat to this may be those patients with greater SUA or tophi at baseline, who are likely to have a higher burden of crystals so are more likely to have a flare when their uric acid level is reduced. However, further specific study comparing these two options is required.

A statistically significant change in the pain domain of the SF-36 shows that with effective treatment of gout a patient's quality of life can improve. This agrees with one previous study ${ }^{27}$ that found a statistically significant improvement in six of the SF-36 domains after 12 months of ULT, with the largest improvement in the bodily pain domain. However, they excluded patients who had had a gout flare in the 4 weeks before completing the questionnaire, which may explain the increased number of significantly improved domains.

There are several caveats to this study. First, although the response rate to the questionnaire was reasonable, not all patients with gout accepted the invitation to take part in the study so there may have been selection bias towards those patients who were more interested in receiving treatment. Second, the duration of the study was only 12 months, which is too short a period to expect complete elimination of urate crystals from all participants and to demonstrate 'cure'. Nevertheless, the mean frequency of attacks was less during the study period than in the previous year and the size and number of tophi in those with subcutaneous deposits was diminishing, suggesting that given longer follow-up all participants with effectively lowered SUA would reach the state of 'cure'. Third, this was a hospitalbased study in which participants were fully assessed by a gout expert within a longer than average initial appointment. Such contextual aspects will have influenced patient expectancy and outcomes, and may limit the generalisability of the findings. However, the year-long follow-up was carried out by a nurse, and nurse-led management of other chronic conditions has been successfully transferred into primary care. ${ }^{15} 16$ Given the excellent outcome such a predominantly nurse-led service could prove cost-effective for the NHS. However, further work would need to ascertain whether a nurse-lead community approach could be successful for gout management, and this is the subject of a recently funded forthcoming randomised controlled trial.

In conclusion, this proof-of-concept study has shown that a predominantly nurse-led complex intervention that includes key elements of recommended best practice is successful in achieving the therapeutic target and maintaining high adherence to ULT over a 12-month period in more than nine out of 10 people with clinically evident gout. Further study is required to determine whether a similar nurse-led intervention can be delivered successfully over a longer period in primary care and result in a higher rate of 'cure' than is currently seen in people with gout.

Acknowledgements The authors are indebted to Arthritis Research UK who funded this work (Award reference 18827) and to the patients who participated in the study. Arthritis Research UK did not participate in study design; in the collection, analysis and interpretation of data; in the writing of the report; or in the decision to submit the article for publication.

Contributors All authors were involved in: conception and design, acquisition of data or analysis and interpretation of data; drafting the article or revising it critically for important intellectual content; final approval of the version published.

Funding Arthritis Research UK.

Competing interests (1) FR, WJ, MD have no support from companies for the submitted work; (2) MD has received consultancy or speaker fees from Ardea Biosciences, Ipsen, Menarini, Novartis and Savient within the past 3 years (3) FR, WJ and MD's spouses, partners, or children have no financial relationships that may be relevant to the submitted work; and (4) FR, WJ, MD have no non-financial interests that may be relevant to the submitted work.

Ethics approval NHS Nottingham County research ethics committee.

Provenance and peer review Not commissioned; externally peer reviewed.

\section{REFERENCES}

1. Mikuls TR, Farrar JT, Bilker WB, et al. Gout epidemiology: results from the UK General Practice Research Database, 1990-1999. Ann Rheum Dis 2005;64:267-72.

2. Lawrence RC, Helmick CG, Arnett FC, et al. Estimates of the prevalence of arthritis and selected musculoskeletal disorders in the United States. Arthritis Rheum 1998;41:778-99.

3. Roddy E, Zhang W, Doherty M. The changing epidemiology of gout. Nat Clin Pract Rheumatol 2007;3:443-9.

4. Perez-Ruiz F. Treating to target: a strategy to cure gout. Rheumatology (Oxford) 2009;48 Suppl 2:iï-iï14.

5. Mikuls TR, Farrar JT, Bilker WB, et al. Suboptimal physician adherence to quality indicators for the management of gout and asymptomatic hyperuricaemia: results from the UK General Practice Research Database (GPRD). Rheumatology (Oxford) 2005; 44:1038-42.

6. Neogi T, Hunter DJ, Chaisson CE, et al. Frequency and predictors of inappropriate management of recurrent gout attacks in a longitudinal study. J Rheumatol 2006;33:104-9

7. Roddy $\mathbf{E}$, Zhang W, Doherty M. Concordance of the management of chronic gout in a UK primary-care population with the EULAR gout recommendations. Ann Rheum Dis 2007;66:1311-15.

8. Annemans L, Spaepen E, Gaskin M, et al. Gout in the UK and Germany: prevalence, comorbidities and management in general practice 2000-2005. Ann Rheum Dis 2008;67:960-6.

9. Dalbeth N, Petrie KJ, House M, et al. Illness perceptions in patients with gout and the relationship with progression of musculoskeletal disability. Arthritis Care Res (Hoboken) 2011;63:1605-12.

10. Solomon DH, Avorn J, Levin R, et al. Uric acid lowering therapy: prescribing patterns in a large cohort of older adults. Ann Rheum Dis 2008;67:609-13.

11. Reach G. Treatment adherence in patients with gout. Joint Bone Spine 2011:78:456-9.

12. Jordan KM, Cameron JS, Snaith M, et al. British Society for Rheumatology and British Health Professionals in Rheumatology guideline for the management of gout. Rheumatology (Oxford) 2007:46:1372-4.

13. Zhang W, Doherty M, Bardin T, et al. EULAR evidence based recommendations for gout. Part II: Management. Report of a task force of the EULAR Standing Committee for International Clinical Studies Including Therapeutics (ESCISIT). Ann Rheum Dis 2006;65:1312-24. 
14. Sutaria S, Katbamna R, Underwood M. Effectiveness of interventions for the treatment of acute and prevention of recurrent gout-a systematic review. Rheumatology (Oxford) 2006;45:1422-31.

15. Griffiths C, Foster G, Barnes N, et al. Specialist nurse intervention to reduce unscheduled asthma care in a deprived multiethnic area: the east London randomised controlled trial for high risk asthma (ELECTRA). BMJ 2004;328:144.

16. Welch G, Garb J, Zagarins S, et al. Nurse diabetes case management interventions and blood glucose control: results of a meta-analysis. Diabetes Res Clin Pract 2010;88:1-6.

17. Zhang $\mathbf{W}$, Doherty $\mathrm{M}$, Pascual $\mathrm{E}$, et al. EULAR evidence based recommendations for gout. Part I: Diagnosis. Report of a task force of the Standing Committee for International Clinical Studies Including Therapeutics (ESCISIT). Ann Rheum Dis 2006;65:1301-11.

18. Gout. Arthritis Research UK, 2011. http://www.arthritisresearchuk.org/ /media/ Files/Arthritis-information/Conditions/2015-Gout.ashx (accessed April 2012).

19. Harrold LR, Andrade SE, Briesacher BA, et al. Adherence with urate-lowering therapies for the treatment of gout. Arthritis Res Ther 2009;11:R46.

20. Sundy JS. Gout management: let's get it right this time. Arthritis Rheum 2008;59:1535-7.
21. Spencer K, Carr A, Doherty M. Patient and provider barriers to effective management of gout in general practice: a qualitative study. Ann Rheum Dis Published Online First 22 March 2012. doi:10.1136/annrheumdis-2011-200801.

22. Lindsay K, Gow P, Vanderpyl J, et al. The experience and impact of living with gout: a study of men with chronic gout using a qualitative grounded theory approach. J Clin Rheumato/ 2011;17:1-6

23. Briesacher BA, Andrade SE, Fouayzi $\mathrm{H}$, et al. Comparison of drug adherence rates among patients with seven different medical conditions. Pharmacotherapy 2008;28:437-43.

24. Febuxostat for the management of hyperuricaemia in people with gout. Technology appraisals, TA164 - Issued: December 2008. National Institute for Health and Clinical Excellence, 2008.

25. Sundy JS, Baraf HS, Yood RA, et al. Efficacy and tolerability of pegloticase for the treatment of chronic gout in patients refractory to conventional treatment: two randomized controlled trials. JAMA 2011;306:711-20.

26. Becker MA, Schumacher HR, Espinoza LR, et al. The urate-lowering efficacy and safety of febuxostat in the treatment of the hyperuricemia of gout: the CONFIRMS trial. Arthritis Res Ther 2010;12:R63.

27. Khanna PP, Perez-Ruiz F, Maranian P, et al. Long-term therapy for chronic gout results in clinically important improvements in the health-related quality of life: short form-36 is responsive to change in chronic gout. Rheumatology (Oxford) 2011;50:740-5. 